\title{
Existence Analysis For a Model Describing Flow of an Incompressible Chemically Reacting Non-Newtonian Fluid
}

\author{
M. Buliček and P. Pustějovská
}

Preprint no. 2013-019 


\title{
EXISTENCE ANALYSIS FOR A MODEL DESCRIBING FLOW OF AN INCOMPRESSIBLE CHEMICALLY REACTING NON-NEWTONIAN FLUID
}

\author{
MIROSLAV BULÍČEK AND PETRA PUSTĚJOVSKÁ
}

\begin{abstract}
We consider a system of PDE's describing steady motions of an incompressible chemically reacting non-Newtonian fluid. The system of governing equations composes of the convection-diffusion equation for concentration and generalized Navier-Stokes equations where the generalized viscosity depends polynomially on the shear rate (the modulus of the symmetric part of the velocity gradient) and the coupling is due dependence of the power-law index on the concentration. This dependence of power-law index on the solution itself causes main difficulties in the analysis of the relevant boundary value problem. We generalize the Lipschitz approximation method and show the existence of a weak solution provided that the minimal value of the power-law exponent is bigger than $d / 2$.
\end{abstract}

\section{INTRODUCTION}

We are interested in developing an existence theory for steady flows of incompressible generalized Navier-Stokes equations, wherein the viscosity is a polynomial function of the shear-rate (the modulus of the symmetric velocity gradient) with the power of polynomial dependence on the concentration coupled with convectiondiffusion equation for concentration. Namely, we study the following system of PDE's

$$
\begin{aligned}
\operatorname{div} \boldsymbol{v} & =0, \\
\operatorname{div}(\boldsymbol{v} \otimes \boldsymbol{v})-\operatorname{div} \boldsymbol{S}(c, \boldsymbol{D} \boldsymbol{v}) & =-\nabla \pi+\boldsymbol{f}, \\
\operatorname{div}(c \boldsymbol{v})-\operatorname{div} \boldsymbol{q}_{c}(c, \nabla c, \boldsymbol{D} \boldsymbol{v}) & =0,
\end{aligned}
$$

that is supposed to be satisfied in an open bounded domain $\Omega \subset \mathbb{R}^{d}(d \geqslant 2)$, where $\boldsymbol{v}: \Omega \rightarrow \mathbb{R}^{d}, \pi: \Omega \rightarrow \mathbb{R}, c: \Omega \rightarrow \mathbb{R}_{+}$are unknown velocity, pressure and concentration fields, respectively. Here $f: \Omega \rightarrow \mathbb{R}^{d}$ represents a given density of the bulk force, $\boldsymbol{D} \boldsymbol{v}$ denotes the symmetric part of the velocity gradient $\nabla \boldsymbol{v}$, i.e., $\boldsymbol{D} \boldsymbol{v}=\frac{1}{2}\left(\nabla \boldsymbol{v}+(\nabla \boldsymbol{v})^{\mathrm{T}}\right)$, and $\boldsymbol{S}(c, \boldsymbol{D} \boldsymbol{v})$ and $\boldsymbol{q}_{c}(c, \nabla c, \boldsymbol{D} \boldsymbol{v})$ are the extra stress tensor

2010 Mathematics Subject Classification. 35Q35,76D03,35Q92,76R50.

Key words and phrases. Sobolev spaces with variable exponent, Lipschitz truncation method, non-Newtonian fluid, existence of weak solution, concentration dependent power-law exponent, convection-diffusion equation.

M. Bulíček thanks to ERC-CZ project LL1202 (MORE) and to Karel Janeček foundation. M. Bulíček is a researcher in the University Center for Mathematical Modeling, Applied Analysis and Computational Mathematics (Math MAC). P. Pustějovská thanks to the Austrian Science Fund (FWF) under the grant SFB Mathematical Optimization and Applications in Biomedical Sciences, and Graz University of Technology. 
of the Cauchy stress tensor and the diffusion flux, respectively. To complete the problem (1.1)-(1.3) we prescribe the following Dirichlet boundary conditions

$$
\boldsymbol{v}=\mathbf{0}, \quad c=c_{d} \quad \text { on } \partial \Omega,
$$

for which we denote $c^{-}:=\min _{x \in \partial \Omega} c_{d}$ and $c^{+}:=\max _{x \in \partial \Omega} c_{d}$. We assume the extra stress tensor $\boldsymbol{S}: \mathbb{R}_{0}^{+} \times \mathbb{R}_{s y m}^{d \times d} \rightarrow \mathbb{R}_{s y m}^{d \times d}$ being a continuous mapping that fulfills following growth, strict monotonicity and coercivity conditions for all $c \in\left[c^{-}, c^{+}\right]$ and all $\boldsymbol{B}, \boldsymbol{B}_{1}, \boldsymbol{B}_{2} \in \mathbb{R}_{\text {sym }}^{d \times d}$

$$
\begin{aligned}
& |\boldsymbol{S}(c, \boldsymbol{B})| \leqslant C_{1}\left(|\boldsymbol{B}|^{p(c)-1}+1\right), \\
& \left(\boldsymbol{S}\left(c, \boldsymbol{B}_{1}\right)-\boldsymbol{S}\left(c, \boldsymbol{B}_{2}\right)\right) \cdot\left(\boldsymbol{B}_{1}-\boldsymbol{B}_{2}\right)>0 \quad \text { for } \boldsymbol{B}_{1} \neq \boldsymbol{B}_{2}, \\
& \boldsymbol{S}(c, \boldsymbol{B}) \cdot \boldsymbol{B} \geqslant C_{2}\left(|\boldsymbol{B}|^{p(c)}+|\boldsymbol{S}|^{p^{\prime}(c)}\right)-C_{3},
\end{aligned}
$$

where $p: \mathbb{R}_{+} \rightarrow \mathbb{R}_{+}$is a Hölder continuous function such that $1<p^{-} \leqslant p(c) \leqslant p^{+}<$ $\infty$ and $p^{\prime}(c)$ is defined as $\frac{p(c)}{p(c)-1}$. Additionally, we assume that the concentration flux vector $\boldsymbol{q}_{c}(c, \boldsymbol{g}, \boldsymbol{B}): \mathbb{R} \times \mathbb{R}^{d} \times \mathbb{R}^{d \times d} \rightarrow \mathbb{R}^{d}$ is a continuous mapping being in addition linear with respect to $\boldsymbol{g}$ and fulfilling for all $c, \boldsymbol{g}, \boldsymbol{B} \in \mathbb{R} \times \mathbb{R}^{d} \times \mathbb{R}^{d \times d}$ the following inequalities

$$
\begin{gathered}
\left|\boldsymbol{q}_{c}(c, \boldsymbol{g}, \boldsymbol{B})\right| \leqslant C_{4}|\boldsymbol{g}|, \\
\boldsymbol{q}_{c}(c, \boldsymbol{g}, \boldsymbol{B}) \cdot \boldsymbol{g} \geqslant C_{5}|\boldsymbol{g}|^{2},
\end{gathered}
$$

where $C_{i}$ are some positive constants.

The prototype examples, we have in mind, are of the following form

$$
\boldsymbol{S}(c, \boldsymbol{D} \boldsymbol{v})=\nu(c,|\boldsymbol{D} \boldsymbol{v}|) \boldsymbol{D} \boldsymbol{v}, \quad \boldsymbol{q}_{c}(c, \nabla c, \boldsymbol{D} \boldsymbol{v})=\boldsymbol{K}(c,|\boldsymbol{D} \boldsymbol{v}|) \nabla c,
$$

where the generalized viscosity $\nu(c,|\boldsymbol{D} \boldsymbol{v}|)$ depends on the shear-rate and on the concentration in the following fashion

$$
\nu(c,|\boldsymbol{D} \boldsymbol{v}|) \sim \nu_{0}\left(\kappa_{1}+\kappa_{2}|\boldsymbol{D} \boldsymbol{v}|^{2}\right)^{\frac{p(c)-2}{2}},
$$

where $\nu_{0}, \kappa_{1}, \kappa_{2}$ stand for positive constants. To $p(\cdot)$ we shall refer to a variable exponent function. Since we have in mind a specific application (as described in the paragraph lower), this function satisfies additional requirements. More precisely, it is a continuous strictly monotone function reflecting shear-thinning/shear-thickening properties of the fluid, this means, it is bounded by $1<p(c)<\infty$, where both limits hold for non-physiological and/or non-physical values of $c$ (either 0 or 1 (infinite concentration)). One example of such function is plotted in Fig. 1.

Such a system is suitable for a description of various biological fluids, e. g. synovial fluid or blood. Even though the rheological responses of both these type of fluids are based on presence of different chemical/biological constituent, they behave as shear-thinning fluids (for example at simple shear test), for which the measure of "how the fluid thins the shear" is related to a concentration of this constituent. In the case of blood, these are the red blood cells (and their ability of network formation), see for example [3] or [18, Chap. 2], on the other hand, in the case of synovial fluid, the governing concentration corresponds to one particular polysaccharide, see for example [20] or [27]. Here, we shall not discuss the rheological background of those fluids in more detail, rather we refer the interested reader to $[3,18,20,27]$ devoted to this topic. 


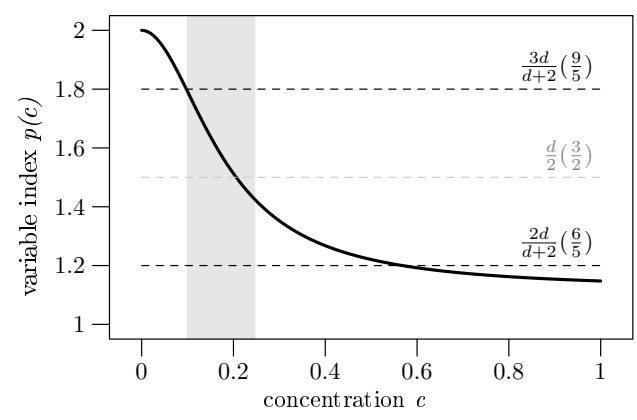

FIgURE 1. Shear-thinning index of viscosity function (1.8) for synovial fluid, see [20] or [27]. The exponent $p$ is plotted as function of concentration. The physiological values (standardly observed for non-pathological synovial fluid) are approximately in range $(0.1,0.25)$, in graph depicted by a gray rectangle. Nondimensionalized concentration $c=1$ refers to (non-physical) $100 \%$ concentration of the solvent. Dashed lines correspond to lower bounds $p^{-}$as required by the mathematical tools employed in the proof of existence of a weak solution. As one can see, bound $p^{-} \geqslant \frac{3 d}{d+2}$ is too restrictive and $p^{-}>\frac{d}{2}$ is unrealizable for higher physiological concentration.

\section{Notation AND the MAin RESUlt}

In this section we introduce the function spaces used in the paper, the basic notation and finally we state the main result. We denote the set of all measurable functions $p: \Omega \rightarrow[1, \infty]$ by $\mathcal{P}(\Omega)$, and call the function $p \in \mathcal{P}(\Omega)$ a variable exponent. Then we define $p^{-}:=\operatorname{ess}_{\inf } \in \Omega(x)$ and $p^{+}:=\operatorname{ess}_{\sup _{x \in \Omega}} p(x)$. Further, for simplicity, we assume only the case when

$$
1<p^{-} \leqslant p^{+}<\infty
$$

We introduce the generalized Lebesgue spaces equipped with corresponding Luxembourg norms

$$
\begin{aligned}
L^{p(\cdot)}(\Omega) & :=\left\{u \in L_{l o c}^{1}(\Omega): \int_{\Omega}|u(x)|^{p(x)} d x<\infty\right\}, \\
\|u\|_{L^{p(\cdot)}(\Omega)}=\|u\|_{p(\cdot)} & :=\inf \left\{\lambda>0: \int_{\Omega}\left|\frac{u(x)}{\lambda}\right|^{p(x)} d x \leqslant 1\right\} .
\end{aligned}
$$

In the same manner we define the generalized Sobolev spaces

$$
\begin{gathered}
W^{1, p(\cdot)}(\Omega):=\left\{u \in W^{1,1}(\Omega) \cap L^{p(\cdot)}(\Omega):|\nabla u| \in L^{p(\cdot)}(\Omega)\right\}, \\
\|u\|_{W^{1, p(\cdot)}(\Omega)}=\|u\|_{1, p(\cdot)}:=\inf \left\{\lambda>0: \int_{\Omega}\left|\frac{u(x)}{\lambda}\right|^{p(x)}+\left|\frac{\nabla u(x)}{\lambda}\right|^{p(x)} d x \leqslant 1\right\} .
\end{gathered}
$$

All above spaces are Banach spaces, and due to (2.1) they are separable and reflexive. Moreover, $\left(L^{p(\cdot)}(\Omega)\right)^{*}=L^{p^{\prime}(\cdot)}(\Omega)$ where the dual variable exponent $p^{\prime} \in \mathcal{P}(\Omega)$ is defined by $\frac{1}{p(x)}+\frac{1}{p^{\prime}(x)}=1$. 
Additionally to the Lebesgue and Sobolev spaces we introduce the function spaces relevant to the problems of incompressible fluids. First, $X(\Omega)^{d}$ denotes space of $d$-vector valued functions with components from $X(\Omega)$. In the same manner we also define the space of tensor-valued functions $X(\Omega)^{d \times d}$. Then we introduce the following notation

$$
\begin{aligned}
W_{0}^{1, p(\cdot)}(\Omega) & :=\left\{u \in W^{1, p(\cdot)}(\Omega): u=0 \text { on } \partial \Omega\right\}, \\
W_{0, \operatorname{div}}^{1, p(\cdot)}(\Omega) & :=\left\{\boldsymbol{u} \in W_{0}^{1, p(\cdot)}(\Omega)^{d}: \operatorname{div} \boldsymbol{u}=0\right\}, \\
L_{0}^{p(\cdot)}(\Omega) & :=\left\{f \in L^{p(\cdot)}(\Omega): \int_{\Omega} f(x) d x=0\right\} .
\end{aligned}
$$

Through the whole text, we denote a duality pairing between $f \in X$ and $g \in X^{\star}$ by $\langle f, g\rangle_{X, X^{\star}}$, or, if it is clear from the context, we skip for simplicity the indices and write $\langle f, g\rangle$. By $\boldsymbol{A} \cdot \boldsymbol{B}$ we denote a scalar product between two tensors, $|Q|$ stands for the Lebesgue measure of the set $Q \subset \mathbb{R}^{d}$, and $C$ refers to some general positive constant (or function independent of the crucial variables) which may change at each appearance.

Now, we are ready to formulate the main theorem on existence of a weak solution to a system (1.1)-(1.7).

Theorem 1. Let $\Omega \subset \mathbb{R}^{d}$ with $d \geqslant 2$ be a bounded Lipschitz domain and $c_{d} \in$ $W^{1, q}(\Omega)$ for some $q>d$. Let us denote

$$
c^{-}:=\min _{x \in \partial \Omega} c_{d}(x), \quad c^{+}:=\max _{x \in \partial \Omega} c_{d}(x)
$$

and assume that $p: \mathbb{R}_{+} \rightarrow \mathbb{R}_{+}$is a Hölder continuous function such that $\frac{d}{2}<p^{-} \leqslant$ $p(c) \leqslant p^{+}<\infty$ for all $c \in\left[c^{-}, c^{+}\right]$. Moreover, let $\boldsymbol{f} \in\left(W_{0, \operatorname{div}}^{1, p^{-}}(\Omega)\right)^{*}$ and $\boldsymbol{S}$ and $\boldsymbol{q}_{c}$ satisfy the assumptions (1.4)-(1.7). Then there exists a couple $(\boldsymbol{v}, c)$ and some $\alpha \in(0,1)$ such that

$$
\begin{aligned}
\left(c-c_{d}\right) & \in \mathcal{C}^{0, \alpha}(\Omega) \cap W_{0}^{1,2}(\Omega), \\
\boldsymbol{v} & \in W_{0, \operatorname{div}}^{1, p(c)}(\Omega),
\end{aligned}
$$

fulfilling

$$
\begin{array}{ll}
\int_{\Omega} \boldsymbol{S}(c, \boldsymbol{D} \boldsymbol{v}) \cdot \nabla \boldsymbol{\psi}-(\boldsymbol{v} \otimes \boldsymbol{v}) \cdot \nabla \boldsymbol{\psi} d x=\langle\boldsymbol{f}, \boldsymbol{\psi}\rangle & \forall \boldsymbol{\psi} \in W_{0, \mathrm{div}}^{1, \infty}(\Omega), \\
\int_{\Omega} \boldsymbol{q}_{c}(c, \nabla c, \boldsymbol{D} \boldsymbol{v}) \cdot \nabla \varphi-c \boldsymbol{v} \cdot \nabla \varphi d x=0 & \forall \varphi \in W_{0}^{1,2}(\Omega) .
\end{array}
$$

The existence analysis for a similar model (even unsteady case) was developed in [4], where however the power-law index is fixed and does not depend on the concentration. Here, we have to deal with a model, where the value of the index is in principle $x$-dependent and in addition a priori unknown. Such a generalization then leads to difficulties in the analysis of the corresponding system of PDE's. To illustrate them, we first recall the results related to our problem where the power-law index is a given number or a given $x$-dependent function. For the fixed $x$-independent exponent, one can use the method of monotone operators provided that $\boldsymbol{\psi}:=\boldsymbol{v}$ in (2.2) is admissible test function. This then naturally leads to the restriction on the class of $p$ 's for such (due to the convective term) $W^{1, p} \hookrightarrow L^{2 p^{\prime}}$, and thus to $p \geqslant \frac{3 d}{d+2}$. Nevertheless, such a bound is unsatisfactory in many application. 
This gave rise to a real interest to lower it to a more realistic one, which led to a series of papers where the final limit of $p>\frac{2 d}{d+2}$ was reached (as far as it seems the most optimal one), corresponding to the compact embedding $W^{1, p} \hookrightarrow \hookrightarrow L^{2}$, and consequently to compactness of the convective term in $L^{1}$. We refer the interested reader to $[13,16,17]$ where the existence theory for fixed index is established and where the so-called $L^{\infty}$ and Lipschitz approximation methods are developed.

Interestingly, it was observed that in many situations the power-law index may not be fixed but can depend also on the spatial variable $x$, see e.g. [28] for a model of generalized Navier-Stokes equations wherein viscosity is of similar power growth as ours but with $p(\cdot):=p\left(|\boldsymbol{E}(x)|^{2}\right) \sim p(x), \boldsymbol{E}$ being a given electric field vector. Such models were studied in $[29,30]$, where the existence theory is built by using the monotone operator theory and also the so-called higher regularity technique under the assumption that the given $\boldsymbol{E}$ and consequently $p$ is smooth enough. Recently, the Lipschitz approximation method was generalized to the spaces with variable exponents in [13], where the existence theory is established if $p^{-}>\frac{2 d}{d+2}$ provided that $p(\cdot)$ is a given log-Hölder continuous function (see the next section for the precise definition).

Finally, the mathematical analysis of the model where the power-law index is also unknown starts in [5], where the existence theory is established with the help of generalized monotone operator theory for $p^{-}>\frac{3 d}{d+2}$. Nevertheless, as Fig. 1 suggests and as we have discussed above, the assumption on the bound of the exponent function arising from the compactness argument is rather too restrictive than realistic. Theorem 1 then gives the final answer for the case when we are able to provide the Hölder continuity of the concentration. We would like to emphasize here, that the bound $p^{-}>\frac{d}{2} \geqslant \frac{2 d}{d+2}$ corresponds to the setting for which one can prove the Hölder continuity of $c$ (and consequently of $p$ ) by the means of De Giorgi method applied on (2.3). Moreover, in view of the results in [13], at least some continuity of the power-law exponent is needed not only for the Lipschitz approximation method, where such a fact is profoundly used, but also from the point of view of the function spaces theory (the Korn inequality, the embedding theorems, the continuity of the maximal function, etc., see the next section for more details). In addition, the technique developed in this paper is a generalization of the Lipschitz approximation

method, where however such approximation is essentially done for a sequence of functions and also for a sequence of Sobolev spaces with variable exponent. We also believe that this may serve as a starting point for further analysis of similar problems.

The rest of the paper is organized as follows. In Section 3 we recall the properties of the Sobolev spaces with variable exponent and also some auxiliary results needed in the proof of the main theorem. Then in Section 4, we prove a generalization of the Lipschitz approximation method and finally in Section 5 we give the proof of Theorem 1.

\section{Auxiliary tools \& Results}

In this section, we introduce all necessary technical tools needed in the paper. First, we introduce a subset $\mathcal{P}^{\log }(\Omega) \subset \mathcal{P}(\Omega)$ as a class of log-Hölder continuous 
exponents satisfying

$$
|p(x)-p(y)| \leqslant \frac{C_{p(\cdot)}}{-\ln |x-y|} \quad \forall x, y \in \Omega: 0<|x-y| \leqslant \frac{1}{2} .
$$

Note that standard Hölder continuous functions on $\Omega$ (which are bounded as required) belong to this class. Also for later purpose, we define for any $u \in L^{1}\left(\mathbb{R}^{d}\right)$ the (non-centered) maximal function $M u$ by

$$
(M u)(x):=\sup _{r>0} \frac{1}{B_{r}(x)} \int_{B_{r}(x)}|u(y)| d y
$$

where the supremum is taken over all balls (cubes) containing $x$, and $M$ is the so-called Hardy-Littlewood maximal operator. Similarly, for $\boldsymbol{u} \in W^{1,1}\left(\mathbb{R}^{d}\right)^{d}$ we denote $M(\boldsymbol{D u}):=M(|\boldsymbol{D} \boldsymbol{u}|)$.

Having this notation we can introduce the first lemma recalling the basic properties of variable Sobolev spaces with log-Hölder continuous exponent.

Lemma 2 (Properties of variable exponent Sobolev spaces, [10]). Let $\Omega \subset \mathbb{R}^{d}$ be an open bounded Lipschitz domain and let $p \in \mathcal{P}^{\log }(\Omega)$ satisfy (2.1). Then we have the density of smooth functions, i.e.,

$$
\overline{\mathcal{C}^{\infty}(\bar{\Omega})} \|^{\|\cdot\|_{1, p(\cdot)}}=W^{1, p(\cdot)}(\Omega),
$$

the embedding theorem, i.e., if $1<p^{-} \leqslant p^{+}<d$ then

$$
W^{1, p(\cdot)}(\Omega) \hookrightarrow L^{q(\cdot)}(\Omega) \quad 1 \leqslant q(x) \leqslant \frac{d p(x)}{d-p(x)}=: p^{*}(x),
$$

being compact for $q(x)<p^{*}(x)$, and the Korn inequality

$$
\|\nabla \boldsymbol{v}\|_{p(\cdot)} \leqslant C\left(\Omega, C_{p(\cdot)}\right)\|\boldsymbol{D} \boldsymbol{v}\|_{p(\cdot)} \quad \text { for all } \boldsymbol{v} \in W_{0}^{1, p(\cdot)}(\Omega)^{d} .
$$

We would like to mention that the book [10] is referred here as a comprehensive source of information. We refer the interested reader to [14, 21, 22, 23, 25, 31] for more detail description and for the original proofs. In addition, note that the log-Hölder continuity is not the necessary assumption for the validity of Lemma 2 but it is known that it is almost the "optimal" property as indicated for example in in [9] or [26]. Moreover, we refer also to [11, 15, 19], works devoted to the difficulties connected with non-validity of Lemma 2 in the context of fluid mechanics.

Next, we recall the extension theorem for variable exponent and the continuity of the maximal function in variable exponent spaces.

Lemma 3 (Variable index extension, [6]). Let $\Omega \subset \mathbb{R}^{d}$ be an open bounded Lipschitz domain and let $p \in \mathcal{P}^{\log }(\Omega)$ be arbitrary. Then there exists an extension ${ }^{1} q \in$ $\mathcal{P}^{\log }\left(\mathbb{R}^{d}\right)$ such that $q^{-}=p^{-}$and $q^{+}=p^{+}$, and the Hardy-Littlewood maximal operator $M$ is continuous from $L^{q(\cdot)}\left(\mathbb{R}^{d}\right)$ to $L^{q(\cdot)}\left(\mathbb{R}^{d}\right)$.

Following result summarizes the properties of Bogovskil operator in the variable exponent settings.

\footnotetext{
${ }^{1}$ For unbounded domains, function from $\mathcal{P}^{\text {log }}$ has to additionally satisfy a proper log-Hölder decay.
} 
Lemma 4 (Bogovskiǔ operator, [10, Sec. 14.3.]). Let $\Omega \subset \mathbb{R}^{d}$ be a bounded Lipschitz domain and $p \in \mathcal{P}^{\log }(\Omega)$ satisfy (2.1). Then there exists a linear continuous operator $\mathcal{B}: L_{0}^{p(\cdot)}(\Omega) \rightarrow W_{0}^{1, p(\cdot)}(\Omega)^{d}$ such that for each $f \in L_{0}^{p(\cdot)}(\Omega)$ we have

$$
\begin{aligned}
\operatorname{div}(\mathcal{B} f) & =f, \\
\|\mathcal{B} f\|_{1, p(\cdot)} & \leqslant C\|f\|_{p(\cdot)},
\end{aligned}
$$

where $C$ depends on $\Omega, p^{-}, p^{+}$and $C_{p(\cdot)}$ from (3.1).

Next, in order to ensure the Hölder continuity of the variable exponent the following celebrated result will be used.

Lemma 5 (De Giorgi [8] - Nash [24], see also [2, Sec. 2.3.]). Let $\Omega \subset \mathbb{R}^{d}$ be a bounded set with Lipschitz boundary and let $q>d$ be given. Assume that that there are $C_{1}, C_{2}>0$ such that

$$
\boldsymbol{K} \in L^{\infty}(\Omega)^{d \times d}: \quad\left|K_{i j}\right| \leqslant C_{1}, \quad \boldsymbol{K} \boldsymbol{b} \cdot \boldsymbol{b} \geqslant C_{2}|\boldsymbol{b}|^{2} \quad \text { for all } \boldsymbol{b} \in \mathbb{R}^{d} .
$$

Then there exists $\alpha>0$ depending only on $\Omega, C_{1}, C_{2}$ and $q$, such that for any $\boldsymbol{g} \in L^{q}(\Omega)^{d}$ and any $c_{d} \in W^{1, q}(\Omega)$ there exists unique $c \in W^{1,2}(\Omega)$ such that $c-c_{d} \in W_{0}^{1,2}(\Omega) \cap \mathcal{C}^{0, \alpha}(\Omega)$ solving

$$
\int_{\Omega} \boldsymbol{K} \nabla c \cdot \nabla \varphi d x=\int_{\Omega} \boldsymbol{g} \cdot \nabla \varphi d x \quad \forall \varphi \in W_{0}^{1,2}(\Omega),
$$

and fulfilling the uniform estimate

$$
\|c\|_{W^{1,2} \cap \mathcal{C}^{0, \alpha}} \leqslant C\left(\Omega, C_{1}, C_{2}, q,\|\boldsymbol{g}\|_{q},\left\|c_{d}\right\|_{1, q}\right) .
$$

Finally, we recall the result on the Lipschitz approximation of functions belonging to $W^{1,1}\left(\mathbb{R}^{d}\right)^{d}$.

Lemma 6 (Lipschitz approximation for $W^{1,1}\left(\mathbb{R}^{d}\right)^{d}$, [1]). There exists $C>0$ depending only on the dimension $d$ such that for all $\boldsymbol{u} \in W^{1,1}\left(\mathbb{R}^{d}\right)^{d}$ and all $\lambda>0$ there exists $\boldsymbol{u}_{\lambda} \in W^{1, \infty}\left(\mathbb{R}^{d}\right)^{d}$ such that

$$
\begin{gathered}
\left\|\boldsymbol{u}_{\lambda}\right\|_{1, \infty} \leqslant C \lambda, \\
\left\{x \in \mathbb{R}^{d}: \boldsymbol{u}(x) \neq \boldsymbol{u}_{\lambda}\right\} \subset\left\{x \in \mathbb{R}^{d}: M(\nabla \boldsymbol{u})(x)>\lambda\right\} .
\end{gathered}
$$

The proof of Lemma 6 is based on the extension theorem. However, a more constructive proof can be found e.g. in [12].

\section{Lipschitz APproximation MEthod IN $W^{1, p^{n}(\cdot)}$}

The use of the Lipschitz approximation method is the heart of the proof of Theorem 1 needed for identification of the weak limit of the extra Cauchy stress. Therefore, we formulate and prove it in this separated section, using the notation of the next Section 5. The couple $\left(\boldsymbol{v}^{n}, p^{n}\right)$ denotes a sequence of approximative solution where

$$
p^{n}(x):=\left(p \circ c^{n}\right)(x) \quad \text { for all } x \in \Omega,
$$

with $c^{n}$ being a sequence of approximative concentrations. The following theorem therefore essentially extends the similar result from [13], where however the authors did not need to face the difficulty that the variable exponent (and consequently the function spaces) changes with the sequence itself. 
Theorem 7 (Lipschitz approximation). Let $\Omega \subset \mathbb{R}^{d}$ be an open bounded Lipschitz domain and assume that $\left\{p^{n}, \boldsymbol{v}^{n}\right\}_{n=1}^{\infty}$ is a sequence such that $1<p^{-} \leqslant p^{n}(x) \leqslant$ $p^{+}<\infty$ for all $x \in \Omega$ and

$$
\begin{array}{ll}
\boldsymbol{v}^{n} \rightarrow \boldsymbol{v} & \text { weakly in } W_{0}^{1, p^{-}}(\Omega)^{d}, \\
p^{n} \rightarrow p & \text { strongly in } \mathcal{C}^{0, \beta}(\bar{\Omega}),
\end{array}
$$

for some $\beta \in(0,1)$. In addition assume that for all $n \in \mathbb{N}$ there holds

$$
\int_{\Omega}\left|\nabla \boldsymbol{v}^{n}\right|^{p^{n}(x)} d x \leqslant C
$$

Then the weak limit $\boldsymbol{v}$ satisfies

$$
\boldsymbol{v} \in W_{0}^{1, p(\cdot)}(\Omega)^{d} .
$$

Moreover, for all $j \in \mathbb{N}$ there exists a sequence $\left\{\lambda_{j}^{n}\right\}_{n=1}^{\infty}$ such that

$$
\left(2^{j}\right)^{2^{j}} \leqslant \lambda_{j}^{n}<\left(2^{j+1}\right)^{2^{j+1}}
$$

and a sequence of truncations $\boldsymbol{v}_{j}^{n} \in W^{1, \infty}(\Omega)^{d}$ such that for all $n, j \in \mathbb{N}$

$$
\left\|\nabla \boldsymbol{v}_{j}^{n}\right\|_{\infty} \leqslant C \lambda_{j}^{n} \leqslant C\left(2^{j+1}\right)^{2^{j+1}} .
$$

Furthermore, we can extract (not relabeled) subsequence from $n$ such that for each $j \in \mathbb{N}$

$$
\begin{array}{lrr}
(4.8) & \boldsymbol{v}_{j}^{n} \rightarrow \boldsymbol{v}_{j} & \text { strongly in } L^{\sigma}(\Omega)^{d} \text { for all } \sigma \in[1, \infty], \\
(4.9) & \boldsymbol{v}_{j}^{n} \rightarrow \boldsymbol{v}_{j} \quad \text { weakly in } W^{1, \sigma}(\Omega)^{d} \text { for all } \sigma \in[1, \infty), \\
(4.10) & \nabla \boldsymbol{v}_{j}^{n} \stackrel{*}{\rightarrow} \nabla \boldsymbol{v}_{j} \quad{ }^{*} \text {-weakly in } L^{\infty}(\Omega)^{d \times d}, \\
\text { where } \boldsymbol{v}_{j} \in W^{1, \infty}(\Omega)^{d} \text {. Moreover, }
\end{array}
$$

$$
\left\|\nabla \boldsymbol{v}_{j}\right\|_{p(\cdot)} \leqslant C \quad \text { and } \quad \boldsymbol{v}_{j} \rightarrow \boldsymbol{v} \text { a.e. in } \Omega \text { as } j \rightarrow \infty .
$$

In addition, extending $\boldsymbol{v}^{n}$ outside $\Omega$ by zero we have

$$
\left\{x \in \Omega: \boldsymbol{v}_{j}^{n} \neq \boldsymbol{v}^{n}\right\} \subset\left\{x \in \Omega: M\left(\nabla \boldsymbol{v}^{n}\right)>\lambda_{j}^{n}\right\},
$$

and for all $n, j \in \mathbb{N}$

$$
\int_{\Omega}\left|\nabla \boldsymbol{v}_{j}^{n} \chi_{\left\{\boldsymbol{v}_{j}^{n} \neq \boldsymbol{v}^{n}\right\}}\right|^{p^{n}(x)} d x \leq C \int_{\Omega}\left|\lambda_{j}^{n} \chi_{\left\{\boldsymbol{v}_{j}^{n} \neq \boldsymbol{v}^{n}\right\}}\right|^{p^{n}(x)} \leqslant \frac{C}{2^{j}} .
$$

Proof. First, the validity of (4.5) directly follows from (4.2)-(4.4) and from weak lower semicontinuity (see [5] for more details). Next, we extend each $\boldsymbol{v}^{n}$ outside $\Omega$ by zero and each $p^{n}$ as in Lemma 3 . Then, we obtain

$$
\begin{array}{ll}
\boldsymbol{v}^{n} \rightarrow \boldsymbol{v} & \text { in } W^{1, p^{-}}\left(\mathbb{R}^{d}\right)^{d}, \\
p^{n} \rightarrow p & \text { in } \mathcal{C}^{0, \beta}\left(\mathbb{R}^{d}\right),
\end{array}
$$

and by continuity of the maximal function and (4.4) we directly get

$$
\int_{\mathbb{R}^{d}}\left|M\left(\nabla \boldsymbol{v}^{n}\right)\right|^{p^{n}(x)} d x \leqslant C \int_{\Omega}\left|\nabla \boldsymbol{v}^{n}\right|^{p^{n}(x)} d x \leqslant C .
$$

Let us for each $j \in \mathbb{N}$ define a sequence $\left\{\theta_{j}^{i}\right\}_{i=2^{j}}^{2^{j+1}-1}$ such that

$$
\theta_{j}^{i}:=\left(2^{j}\right)^{i},
$$


and a sequence of subsets $\left\{U_{j, n}^{i}\right\}_{i=2^{j}}^{2^{j+1}-1} \subset \mathbb{R}^{d}$ as

$$
U_{j, n}^{i}:=\left\{x \in \mathbb{R}^{d}: \theta_{j}^{i}<M\left(\nabla \boldsymbol{v}^{n}\right)(x) \leqslant \theta_{j}^{i+1}\right\}
$$

Note, that $U_{j, n}^{i}$ are mutually disjoint bounded sets, and thus

$$
\sum_{i=2^{j}}^{2^{j+1}-1} \int_{U_{j, n}^{i}}\left|M\left(\nabla \boldsymbol{v}^{n}\right)\right|^{p^{n}(x)} d x \leqslant \int_{\mathbb{R}^{d}}\left|M\left(\nabla \boldsymbol{v}^{n}\right)\right|^{p^{n}(x)} d x \leqslant C .
$$

Since the sum above is formed of $2^{j}$ summands, for each $n$ there must exist $i^{*}$ such that

$$
\int_{U_{j, n}^{i *}}\left|M\left(\nabla \boldsymbol{v}^{n}\right)\right|^{p^{n}(x)} d x \leqslant \frac{C}{2^{j}}
$$

Then, for this $i^{*}$ we set

$$
\lambda_{j}^{n}:=\theta_{j}^{i^{*}}=\left(2^{j}\right)^{i^{*}}
$$

and thus (4.6) follows. This directly gives

$$
\underset{\left\{\lambda_{j}^{n}<M\left(\nabla \boldsymbol{v}^{n}\right) \leqslant 2^{j} \lambda_{j}^{n}\right\}}{\int}\left|M\left(\nabla \boldsymbol{v}^{n}\right)\right|^{p^{n}(x)} d x \leqslant \frac{C}{2^{j}} .
$$

Having such $\lambda_{j}^{n}$ we use Lemma 6 with $\lambda=\lambda_{j}^{n}$ applied on $\boldsymbol{v}^{n}$, thus we introduce

$$
\boldsymbol{v}_{j}^{n}:=\boldsymbol{v}_{\lambda_{j}^{n}}^{n}
$$

From Lemma 6 then directly follow the properties (4.7) and (4.12). To prove (4.13) we use (4.7) and (4.15), hence

$$
\begin{aligned}
\int_{\left\{\boldsymbol{v}^{n} \neq \boldsymbol{v}_{j}^{n}\right\}}\left|\nabla \boldsymbol{v}_{j}^{n}\right|^{p^{n}(x)} d x & \leqslant C \int_{\left\{\boldsymbol{v}^{n} \neq \boldsymbol{v}_{j}^{n}\right\}}\left|\lambda_{j}^{n}\right|^{p^{n}(x)} d x \leqslant \underset{\left\{\lambda_{j}^{n}<M\left(\nabla \boldsymbol{v}^{n}\right)\right\}}{C \int_{U_{j, n}^{i^{*}}}\left|\lambda_{j}^{n}\right|^{p^{n}(x)} d x} \\
& =C \int_{\left\{2^{j} \lambda_{j}^{n}<M\left(\nabla \boldsymbol{v}^{n}\right)\right\}} d x+\quad C \int_{j}^{p^{n}(x)} \mid \lambda^{p^{n}(x)} d x \\
& \leqslant C \int_{U_{j, n}^{i^{*}}}\left(M\left(\nabla \boldsymbol{v}^{n}\right)\right)^{p^{n}(x)} d x+C \int_{\mathbb{R}^{d}}\left(\frac{M\left(\nabla \boldsymbol{v}^{n}\right)}{2^{j}}\right)^{p^{n}(x)} d x \\
& \leqslant \frac{C}{2^{j}}+\frac{C}{\left(2^{j}\right)^{p^{-}}} \int_{\mathbb{R}^{d}}\left(M\left(\nabla \boldsymbol{v}^{n}\right)\right)^{p^{n}(x)} d x \leqslant \frac{C}{2^{j}} .
\end{aligned}
$$

Due to compact embedding, (4.7) and the fact that $\boldsymbol{v}_{j}^{n}$ are compactly supported in $\mathbb{R}^{d}$, we can for any fixed $j \in \mathbb{N}$ extract a subsequence fulfilling (4.8)-(4.10). Moreover, using a diagonal procedure, we can extract another subsequence in $n$ such that (4.8)-(4.10) hold for each $j \in \mathbb{N}$ and $n$ form the extracted indices. Finally, 
it follows from (4.8), (4.2), (4.12) and the Hölder inequality that

$$
\begin{aligned}
\left\|\boldsymbol{v}_{j}-\boldsymbol{v}\right\|_{1} & =\lim _{n \rightarrow \infty} \int_{\Omega}\left|\boldsymbol{v}_{j}^{n}-\boldsymbol{v}^{n}\right| d x \leqslant C \limsup _{n \rightarrow \infty}\left|\left\{\boldsymbol{v}_{j}^{n} \neq \boldsymbol{v}^{n}\right\}\right|^{\frac{1}{p^{-1}}} \\
& \leqslant C \limsup _{n \rightarrow \infty}\left|\left\{M\left(\nabla \boldsymbol{v}^{n}\right)>\lambda_{j}^{n}\right\}\right|^{\frac{1}{p^{-1}}} \leqslant C \limsup _{n \rightarrow \infty}\left(\int_{\Omega} \frac{M\left(\nabla \boldsymbol{v}^{n}\right)}{\lambda_{j}^{n}} d x\right)^{\frac{1}{p^{-1}}} \\
& \leqslant \frac{C}{\left(\lambda_{j}^{n}\right)^{\frac{1}{p^{-1}}}} \leqslant \frac{C}{\left(2^{j}\right)^{\frac{2^{j}}{p^{-1}}}} \leqslant \frac{C}{2^{j}} .
\end{aligned}
$$

Consequently, the second part of (4.11) follows (again for not relabeled subsequence), hence, from the uniqueness of a weak limit, we also have

$$
\boldsymbol{v}_{j} \rightarrow \boldsymbol{v} \quad \text { in } W^{1, p(\cdot)}(\Omega)^{d} .
$$

\section{Proof of the Main theorem}

We start the proof of the main theorem by defining an auxiliary cut-off function. For arbitrary $k>0$ we introduce a smooth function $G_{k}: \mathbb{R}_{0}^{+} \rightarrow[0,1]$ with uniformly ( $k$-independently) bounded derivative such that

$$
G_{k}(s):= \begin{cases}1 & s \leqslant k, \\ 0 & s \geqslant 2 k .\end{cases}
$$

We look for an approximative solution $\left(\boldsymbol{v}^{n}, c^{n}\right)$ such that $\boldsymbol{v}^{n} \in W_{0, \operatorname{div}}^{1, p^{n}(\cdot)}(\Omega)$ (with $p^{n}$ defined as in (4.1)) and $c^{n}-c_{d} \in W_{0}^{1,2}(\Omega)$ such that for all $\boldsymbol{\psi} \in W_{0, \operatorname{div}}^{1, p^{n}(\cdot)}(\Omega)$ and all $\varphi \in W_{0}^{1,2}(\Omega)$

$$
\begin{gathered}
-\int_{\Omega} G_{n}\left(\left|\boldsymbol{v}^{n}\right|^{2}\right)\left(\boldsymbol{v}^{n} \otimes \boldsymbol{v}^{n}\right) \cdot \nabla \boldsymbol{\psi} d x+\int_{\Omega} \boldsymbol{S}\left(c^{n}, \boldsymbol{D} \boldsymbol{v}^{n}\right) \cdot \boldsymbol{D} \boldsymbol{\psi} d x=\langle\boldsymbol{f}, \boldsymbol{\psi}\rangle \\
-\int_{\Omega} c^{n} \boldsymbol{v}^{n} \cdot \nabla \varphi d x+\int_{\Omega} \boldsymbol{q}_{c}\left(c^{n}, \nabla c^{n}, \boldsymbol{D} \boldsymbol{v}^{n}\right) \cdot \nabla \varphi d x=0 .
\end{gathered}
$$

Due to the boundedness of $G_{n}\left(\left|\boldsymbol{v}^{n}\right|^{2}\right)$, one can adapt the technique from [5] (a generalization of the monotone operator theory) and establish the existence of a weak solution to (5.1)-(5.2). Moreover, one can set $\boldsymbol{\psi}:=\boldsymbol{v}^{n}$ in (5.1) and $\varphi:=c^{n}-c_{d}$ in (5.2) and with the help of the assumptions (1.4)-(1.7) and the fact that $\operatorname{div} \boldsymbol{v}^{n}=0$ deduce the following uniform estimate

$$
\int_{\Omega}\left|\boldsymbol{D} \boldsymbol{v}^{n}\right|^{p^{n}}+\left|\boldsymbol{S}\left(c^{n}, \boldsymbol{D} \boldsymbol{v}^{n}\right)\right|^{\left(p^{n}\right)^{\prime}}+\left|\nabla c^{n}\right|^{2}+\left|\boldsymbol{q}_{c}\left(c^{n}, \nabla c^{n}, \boldsymbol{D} \boldsymbol{v}^{n}\right)\right|^{2} d x \leqslant C .
$$

Additionally, since $p^{-}>\frac{d}{2}$ it follows from the embedding theorem, the Korn inequality (used for the standard Sobolev space $W^{1, p^{-}}$) and Lemma 5 that there is $\alpha>0$ such that

$$
\left\|c^{n}\right\|_{0, \alpha} \leqslant C .
$$

Consequently, using (5.3)-(5.4), the reflexivity of the relevant spaces, the Korn inequality and the embedding theorem we can extract subsequences (which we do 
not relabel) such that

$$
\begin{aligned}
& \boldsymbol{v}^{n} \rightarrow \boldsymbol{v} \quad \text { weakly in } W_{0, \operatorname{div}}^{1, p^{-}}(\Omega), \\
& \boldsymbol{v}^{n} \rightarrow \boldsymbol{v} \quad \text { strongly in } L^{2+\varepsilon}(\Omega)^{d},(\varepsilon>0) \text {, } \\
& c^{n} \rightarrow c \quad \text { weakly in } W^{1,2}(\Omega), \\
& c^{n} \rightarrow c \quad \text { strongly in } \mathcal{C}^{0, \tilde{\alpha}}(\Omega),(\tilde{\alpha}<\alpha), \\
& \boldsymbol{S}\left(c^{n}, \boldsymbol{D} \boldsymbol{v}^{n}\right) \rightarrow \overline{\boldsymbol{S}} \quad \text { weakly in } L^{\left(p^{+}\right)^{\prime}}(\Omega)^{d \times d}, \\
& \boldsymbol{q}_{c}\left(c^{n}, \nabla c^{n}, \boldsymbol{D} \boldsymbol{v}^{n}\right) \rightarrow \overline{\boldsymbol{q}}_{c} \quad \text { weakly in } L^{2}(\Omega)^{d} .
\end{aligned}
$$

In addition, following [5], we can show by the weak lower semicontinuity that (5.3) together with the Korn inequality imply

$$
\int_{\Omega}|\nabla \boldsymbol{v}|^{p(x)}+|\overline{\boldsymbol{S}}|^{p^{\prime}(x)} d x \leqslant C .
$$

Having (5.5)-(5.11) and using the fact that $G_{n} \nearrow 1$, we can let $n \rightarrow \infty$ in (5.1)-(5.2) to obtain

$$
\begin{array}{cl}
-\int_{\Omega}(\boldsymbol{v} \otimes \boldsymbol{v}) \cdot \nabla \boldsymbol{\psi} d x+\int_{\Omega} \overline{\boldsymbol{S}} \cdot \boldsymbol{D} \boldsymbol{\psi} d x=\langle\boldsymbol{f}, \boldsymbol{\psi}\rangle & \boldsymbol{\psi} \in W_{0, \mathrm{div}}^{1, \infty}(\Omega), \\
-\int_{\Omega} c \boldsymbol{v} \cdot \nabla \varphi d x+\int_{\Omega} \overline{\boldsymbol{q}}_{c} \cdot \nabla \varphi d x=0 & \varphi \in W_{0}^{1,2}(\Omega) .
\end{array}
$$

Thus, what remains to prove is to show that

$$
\overline{\boldsymbol{S}}=\boldsymbol{S}(c, \boldsymbol{D} \boldsymbol{v}) \quad \text { and } \quad \overline{\boldsymbol{q}}_{c}=\boldsymbol{K}(c, \boldsymbol{D} \boldsymbol{v}) \nabla c .
$$

5.1. Compactness of $\boldsymbol{D} \boldsymbol{v}^{n}$. The first step in proving (5.13) is to show the compactness of $\boldsymbol{D} \boldsymbol{v}^{n}$. Inspired by [7], we aim in this subsection to show that

$$
\lim _{n \rightarrow \infty} \int_{\Omega}\left(\left(\boldsymbol{S}\left(c^{n}, \boldsymbol{D} \boldsymbol{v}^{n}\right)-\boldsymbol{S}\left(c^{n}, \boldsymbol{D} \boldsymbol{v}\right)\right) \cdot\left(\boldsymbol{D} \boldsymbol{v}^{n}-\boldsymbol{D} \boldsymbol{v}\right)\right)^{\frac{1}{4}} d x=0 .
$$

For sure, it follows from (5.3) and the Hölder inequality that

$$
\limsup _{n \rightarrow \infty} \int_{\Omega}\left(\left(\boldsymbol{S}\left(c^{n}, \boldsymbol{D} \boldsymbol{v}^{n}\right)-\boldsymbol{S}\left(c^{n}, \boldsymbol{D} \boldsymbol{v}\right)\right) \cdot\left(\boldsymbol{D} \boldsymbol{v}^{n}-\boldsymbol{D} \boldsymbol{v}\right)\right)^{\frac{1}{4}} d x=L<\infty .
$$

Hence, we need to show $L=0$. Let us for arbitrary fixed $\varkappa>0$ define

$$
\Omega_{\varkappa}:=\{x \in \Omega:|\boldsymbol{D} \boldsymbol{v}|>\varkappa\} \cup\left\{x \in \Omega: \operatorname{dist}(x, \partial \Omega) \leqslant \frac{1}{\varkappa}\right\} .
$$

Note, that our Lipschitz approximations do not vanish on the boundary, and thus we include to the "bad part" of $\Omega$ the second term of the union above. Then, since $\Omega$ is Lipschitz bounded, we have

$$
\left|\Omega_{\varkappa}\right| \leqslant \int_{\Omega} \frac{|\boldsymbol{D} \boldsymbol{v}|}{\varkappa} d x+\frac{C}{\varkappa} \leqslant \frac{C}{\varkappa},
$$

where the last inequality follows from (5.11). Next, we decompose the claimed integral into

$$
\int_{\Omega}\left(\left(\boldsymbol{S}\left(c^{n}, \boldsymbol{D} \boldsymbol{v}^{n}\right)-\boldsymbol{S}\left(c^{n}, \boldsymbol{D} \boldsymbol{v}\right)\right) \cdot\left(\boldsymbol{D} \boldsymbol{v}^{n}-\boldsymbol{D} \boldsymbol{v}\right)\right)^{\frac{1}{4}} d x=J_{\varkappa}^{n}+I_{\varkappa}^{n},
$$


where

$$
\begin{aligned}
& J_{\varkappa}^{n}:=\int_{\Omega_{\varkappa}}\left(\left(\boldsymbol{S}\left(c^{n}, \boldsymbol{D} \boldsymbol{v}^{n}\right)-\boldsymbol{S}\left(c^{n}, \boldsymbol{D} \boldsymbol{v}\right)\right) \cdot\left(\boldsymbol{D} \boldsymbol{v}^{n}-\boldsymbol{D} \boldsymbol{v}\right)\right)^{\frac{1}{4}} d x, \\
& I_{\varkappa}^{n}:=\int_{\Omega \backslash \Omega_{\varkappa}}\left(\left(\boldsymbol{S}\left(c^{n}, \boldsymbol{D} \boldsymbol{v}^{n}\right)-\boldsymbol{S}\left(c^{n}, \boldsymbol{D} \boldsymbol{v}\right)\right) \cdot\left(\boldsymbol{D} \boldsymbol{v}^{n}-\boldsymbol{D} \boldsymbol{v}\right)\right)^{\frac{1}{4}} d x .
\end{aligned}
$$

To estimate $J_{\varkappa}^{n}$, we use (5.3), (5.11) and the Hölder inequality to conclude

$$
J_{\varkappa}^{n} \leqslant C\left|\Omega_{\varkappa}\right|^{\frac{1}{2}} \leqslant \frac{C}{\sqrt{\varkappa}}
$$

Next, we introduce a matrix-truncation function $\boldsymbol{T}_{\varkappa}: \mathbb{R}^{d \times d} \rightarrow \mathbb{R}^{d \times d}$ at height $\varkappa>0$ as

$$
\boldsymbol{T}_{\varkappa}(\boldsymbol{B}):= \begin{cases}\boldsymbol{B} & |\boldsymbol{B}| \leqslant \varkappa, \\ \varkappa \frac{B}{|\boldsymbol{B}|} & |\boldsymbol{B}|>\varkappa .\end{cases}
$$

and rewrite the integral $I_{\varkappa}^{n}$ in the terms of truncation $\boldsymbol{T}_{\varkappa}(\boldsymbol{D} \boldsymbol{v})$ which collides with $\boldsymbol{D} \boldsymbol{v}$ on $\Omega \backslash \Omega_{\varkappa}$, and then, due to positiveness of the argument, we extend the integral on the whole domain, i.e.,

$$
\begin{aligned}
I_{\varkappa}^{n} & =\int_{\Omega \backslash \Omega_{\varkappa}}\left(\left(\boldsymbol{S}\left(c^{n}, \boldsymbol{D} \boldsymbol{v}^{n}\right)-\boldsymbol{S}\left(c^{n}, \boldsymbol{T}_{\varkappa}(\boldsymbol{D} \boldsymbol{v})\right)\right) \cdot\left(\boldsymbol{D} \boldsymbol{v}^{n}-\boldsymbol{T}_{\varkappa}(\boldsymbol{D} \boldsymbol{v})\right)\right)^{\frac{1}{4}} d x \\
& \leqslant \int_{\Omega}\left(\xi_{\varkappa}\left(\boldsymbol{S}\left(c^{n}, \boldsymbol{D} \boldsymbol{v}^{n}\right)-\boldsymbol{S}\left(c^{n}, \boldsymbol{T}_{\varkappa}(\boldsymbol{D} \boldsymbol{v})\right)\right) \cdot\left(\boldsymbol{D} \boldsymbol{v}^{n}-\boldsymbol{T}_{\varkappa}(\boldsymbol{D} \boldsymbol{v})\right)\right)^{\frac{1}{4}} d x,
\end{aligned}
$$

where $\xi_{\varkappa} \in \mathcal{D}(\Omega)$ is arbitrary fixed function such that $0 \leqslant \xi_{\varkappa} \leqslant 1, \xi_{\varkappa}(x)=1$ if $\operatorname{dist}(x, \partial \Omega) \geqslant \frac{1}{x}$. Let us estimate the last term using the Lipschitz truncations. Since $p$ is a Hölder continuous function and $c^{n}$ fulfills (5.8), we see that $\left(\boldsymbol{v}^{n}, p^{n}\right)$ satisfies the assumptions of Theorem 7 and therefore for arbitrary $j \in \mathbb{N}$ we can find $\boldsymbol{v}_{j}^{n} \in W^{1, \infty}(\Omega)^{d}$. Then, by using the Hölder inequality, we have

$$
\begin{aligned}
I_{\varkappa}^{n} & \leqslant \int_{\Omega}\left(\xi_{\varkappa}\left(\boldsymbol{S}\left(c^{n}, \boldsymbol{D} \boldsymbol{v}^{n}\right)-\boldsymbol{S}\left(c^{n}, \boldsymbol{T}_{\varkappa}(\boldsymbol{D} \boldsymbol{v})\right)\right) \cdot\left(\boldsymbol{D} \boldsymbol{v}^{n}-\boldsymbol{T}_{\varkappa}(\boldsymbol{D} \boldsymbol{v})\right)\right)^{\frac{1}{4}} d x \\
& \leqslant\left(\int_{\left\{\boldsymbol{v}_{j}^{n}=\boldsymbol{v}^{n}\right\}} \xi_{\varkappa}\left(\boldsymbol{S}\left(c^{n}, \boldsymbol{D} \boldsymbol{v}^{n}\right)-\boldsymbol{S}\left(c^{n}, \boldsymbol{T}_{\varkappa}(\boldsymbol{D} \boldsymbol{v})\right)\right) \cdot\left(\boldsymbol{D} \boldsymbol{v}_{j}^{n}-\boldsymbol{T}_{\varkappa}(\boldsymbol{D} \boldsymbol{v})\right) d x\right)^{\frac{1}{4}}|\Omega|^{\frac{3}{4}} \\
& +\left(\int_{\left\{\boldsymbol{v}_{j}^{n} \neq \boldsymbol{v}^{n}\right\}}\left(\left(\boldsymbol{S}\left(c^{n}, \boldsymbol{D} \boldsymbol{v}^{n}\right)-\boldsymbol{S}\left(c^{n}, \boldsymbol{T}_{\varkappa}(\boldsymbol{D} \boldsymbol{v})\right)\right) \cdot\left(\boldsymbol{D} \boldsymbol{v}^{n}-\boldsymbol{T}_{\varkappa}(\boldsymbol{D} \boldsymbol{v})\right)\right)^{\frac{1}{2}} d x\right)^{\frac{1}{2}}\left|\left\{\boldsymbol{v}_{j}^{n} \neq \boldsymbol{v}^{n}\right\}\right|^{\frac{1}{2}} \\
& =:\left(I_{\varkappa, j\{=\}}^{n}\right)^{\frac{1}{4}}|\Omega|^{\frac{3}{4}}+\left(I_{\varkappa, j\{\neq\}}^{n}\right)^{\frac{1}{2}}\left|\left\{\boldsymbol{v}_{j}^{n} \neq \boldsymbol{v}^{n}\right\}\right|^{\frac{1}{2}} .
\end{aligned}
$$

First, as an easier term, we handle the latter term with $I_{\varkappa, j\{\neq\}}^{n}$. Note that by (4.6), (4.12) and (4.14) we have

$$
\left|\left\{\boldsymbol{v}_{j}^{n} \neq \boldsymbol{v}^{n}\right\}\right|=\left\|\chi_{\left\{\boldsymbol{v}_{j}^{n} \neq \boldsymbol{v}^{n}\right\}}\right\|_{1, \Omega} \leqslant \int_{\mathbb{R}^{d}} \frac{M\left(\boldsymbol{D} \boldsymbol{v}^{n}\right)}{\lambda_{j}^{n}} d x \leqslant \frac{C}{\left(2^{j}\right)^{2^{j}}},
$$


and thus, it follows from the Hölder inequality, (5.3) and (5.11) that

$$
\left(I_{\varkappa, j\{\neq\}}^{n}\right)^{\frac{1}{2}}\left|\left\{\boldsymbol{v}_{j}^{n} \neq \boldsymbol{v}^{n}\right\}\right|^{\frac{1}{2}} \leqslant \frac{C}{2^{j}} .
$$

Finally, we estimate the first integral $I_{\varkappa, j\{=\}}^{n}$, decomposed into

$$
\begin{aligned}
I_{\varkappa, j\{=\}}^{n} & =\int_{\Omega} \xi_{\varkappa}\left(\boldsymbol{S}\left(c^{n}, \boldsymbol{D} \boldsymbol{v}^{n}\right)-\boldsymbol{S}\left(c^{n}, \boldsymbol{T}_{\varkappa}(\boldsymbol{D} \boldsymbol{v})\right)\right) \cdot\left(\boldsymbol{D} \boldsymbol{v}_{j}^{n}-\boldsymbol{T}_{\varkappa}(\boldsymbol{D} \boldsymbol{v})\right) d x \\
& -\int_{\left\{\boldsymbol{v}_{j}^{n} \neq \boldsymbol{v}^{n}\right\}} \xi_{\varkappa}\left(\boldsymbol{S}\left(c^{n}, \boldsymbol{D} \boldsymbol{v}^{n}\right)-\boldsymbol{S}\left(c^{n}, \boldsymbol{T}_{\varkappa}(\boldsymbol{D} \boldsymbol{v})\right)\right) \cdot\left(\boldsymbol{D} \boldsymbol{v}_{j}^{n}-\boldsymbol{T}_{\varkappa}(\boldsymbol{D} \boldsymbol{v})\right) d x .
\end{aligned}
$$

Let us analyze the second term using the Young inequality, (1.4), and (4.13)

$$
\begin{aligned}
& \left|\int_{\left\{\boldsymbol{v}_{j}^{n} \neq \boldsymbol{v}^{n}\right\}} \xi_{\varkappa}\left(\boldsymbol{S}\left(c^{n}, \boldsymbol{D} \boldsymbol{v}^{n}\right)-\boldsymbol{S}\left(c^{n}, \boldsymbol{T}_{\varkappa}(\boldsymbol{D} \boldsymbol{v})\right)\right) \cdot\left(\boldsymbol{D} \boldsymbol{v}_{j}^{n}-\boldsymbol{T}_{\varkappa}(\boldsymbol{D} \boldsymbol{v})\right) d x\right| \\
& \leqslant \int_{\left\{\boldsymbol{v}_{j}^{n} \neq \boldsymbol{v}^{n}\right\}}\left|\boldsymbol{S}\left(c^{n}, \boldsymbol{D} \boldsymbol{v}^{n}\right) \cdot \boldsymbol{D} \boldsymbol{v}_{j}^{n}\right| d x+C(\varkappa) \int_{\left\{\boldsymbol{v}_{j}^{n} \neq \boldsymbol{v}^{n}\right\}}\left(\left|\boldsymbol{S}\left(c^{n}, \boldsymbol{D} \boldsymbol{v}^{n}\right)\right|+\left|\boldsymbol{D} \boldsymbol{v}_{j}^{n}\right|+1\right) d x \\
& \leqslant C \int_{\left\{\boldsymbol{v}_{j}^{n} \neq \boldsymbol{v}^{n}\right\}}\left|\nabla \boldsymbol{v}^{n}\right|^{p^{n}(x)-1} \lambda_{j}^{n} d x+C(\varkappa)\left|\left\{\boldsymbol{v}_{j}^{n} \neq \boldsymbol{v}^{n}\right\}\right|^{\frac{1}{p^{+}}} \\
& \leqslant \frac{C}{\left(p^{+}\right)^{\prime}} \int_{\left\{\boldsymbol{v}_{j}^{n} \neq \boldsymbol{v}^{n}\right\}}\left|\nabla \boldsymbol{v}^{n}\right|^{p^{n}(x)} d x+\frac{C}{p_{\left\{\boldsymbol{v}_{j}^{n} \neq \boldsymbol{v}^{n}\right\}}} \int_{j}\left|\lambda_{j}^{n}\right|^{p^{n}(x)} d x+\frac{C(\varkappa)}{\left(\lambda_{j}^{n}\right)^{\frac{1}{p^{+}}}} \leqslant \frac{C(\varkappa)}{2^{j}} .
\end{aligned}
$$

To analyze the first term in (5.16), we have no suitable estimates by hand. A natural choice would be, since we can control the convective term for Lipschitz test functions, to use the weak formulation. Here, nevertheless, one can not use the Lipschitz approximation $\boldsymbol{v}_{j}^{n}$ since they do not posses (in general) the divergence-free property, and they do not vanish on the boundary. To elude this fact, let us introduce div-free approximations with zero trace by the means of Bogovskil operator (Proposition 4). First define

$$
\begin{aligned}
\boldsymbol{\zeta}_{\varkappa, j}^{n, 1} & :=\mathcal{B}\left(\xi_{\varkappa} \operatorname{div} \boldsymbol{v}_{j}^{n}-f_{\Omega} \xi_{\varkappa} \operatorname{div} \boldsymbol{v}_{j}^{n} d x\right), \\
\boldsymbol{\zeta}_{\varkappa, j}^{n, 2} & :=\mathcal{B}\left(\nabla \xi_{\varkappa} \cdot \boldsymbol{v}_{j}^{n}-f_{\Omega} \nabla \xi_{\varkappa} \cdot \boldsymbol{v}_{j}^{n} d x\right) .
\end{aligned}
$$

Then, from the linearity of $\mathcal{B}$ it follows

$$
\boldsymbol{\zeta}_{\varkappa, j}^{n}:=\xi_{\varkappa} \boldsymbol{v}_{j}^{n}-\boldsymbol{\zeta}_{\varkappa, j}^{n, 1}-\boldsymbol{\zeta}_{\varkappa, j}^{n, 2}=\xi_{\varkappa} \boldsymbol{v}_{j}^{n}-\mathcal{B}\left(\operatorname{div}\left(\xi_{\varkappa} \boldsymbol{v}_{j}^{n}\right)\right) .
$$

Consequently, from Lemma 4 , it directly follows that $\operatorname{div} \boldsymbol{\zeta}_{\varkappa, j}^{n}=0$ and that $\boldsymbol{\zeta}_{\varkappa, j}^{n}$ has zero trace on $\partial \Omega$. Moreover, from the fact that continuous operator preserves weak convergence, (3.2) and (4.8)-(4.9) it follows that for each $j \in \mathbb{N}$

$$
\begin{aligned}
& \boldsymbol{\zeta}_{\varkappa, j}^{n} \rightarrow \xi_{\varkappa} \boldsymbol{v}_{j}-\mathcal{B}\left(\operatorname{div}\left(\xi_{\varkappa} \boldsymbol{v}_{j}\right)\right) \equiv \boldsymbol{\zeta}_{\varkappa, j} \quad \text { in } W^{1, \sigma}(\Omega)^{d}, \\
& \boldsymbol{\zeta}_{\varkappa, j}^{n} \rightarrow \boldsymbol{\zeta}_{\varkappa, j} \quad \text { in } L^{\sigma}(\Omega)^{d} \text {, } \\
& \boldsymbol{\zeta}_{\varkappa, j}^{n, 2} \rightarrow \mathcal{B}\left(\nabla \xi_{\varkappa} \cdot \boldsymbol{v}_{j}-f_{\Omega} \nabla \xi_{\varkappa} \cdot \boldsymbol{v}_{j} d x\right) \equiv \boldsymbol{\zeta}_{\varkappa, j}^{2} \quad \text { in } W^{1, \sigma}(\Omega)^{d},
\end{aligned}
$$


as $n \rightarrow \infty$, where $\sigma \in(1, \infty)$ is arbitrary. Then, we can rewrite the first term in integral (5.16) in the terms of these approximations to obtain

$$
\begin{aligned}
& \int_{\Omega} \xi_{\varkappa}\left(\boldsymbol{S}\left(c^{n}, \boldsymbol{D} \boldsymbol{v}^{n}\right)-\boldsymbol{S}\left(c^{n}, \boldsymbol{T}_{\varkappa}(\boldsymbol{D} \boldsymbol{v})\right)\right) \cdot\left(\boldsymbol{D} \boldsymbol{v}_{j}^{n}-\boldsymbol{T}_{\varkappa}(\boldsymbol{D} \boldsymbol{v})\right) d x \\
& =\int_{\Omega} \boldsymbol{S}\left(c^{n}, \boldsymbol{D} \boldsymbol{v}^{n}\right) \cdot\left(\boldsymbol{D} \boldsymbol{\zeta}_{\varkappa, j}^{n}+\boldsymbol{D} \boldsymbol{\zeta}_{\varkappa, j}^{n, 2}-\nabla \xi_{\varkappa} \boldsymbol{v}_{j}^{n}\right) d x \\
& +\int_{\Omega} \boldsymbol{S}\left(c^{n}, \boldsymbol{D} \boldsymbol{v}^{n}\right) \cdot \boldsymbol{D} \boldsymbol{\zeta}_{\varkappa, j}^{n, 1} d x \\
& -\int_{\Omega} \xi_{\varkappa} \boldsymbol{S}\left(c^{n}, \boldsymbol{D} \boldsymbol{v}^{n}\right) \cdot \boldsymbol{T}_{\varkappa}(\boldsymbol{D} \boldsymbol{v}) d x-\int_{\Omega} \xi_{\varkappa} \boldsymbol{S}\left(c^{n}, \boldsymbol{T}_{\varkappa}(\boldsymbol{D} \boldsymbol{v})\right) \cdot\left(\boldsymbol{D} \boldsymbol{v}_{j}^{n}-\boldsymbol{T}_{\varkappa}(\boldsymbol{D} \boldsymbol{v})\right) d x \\
& =: Y_{\varkappa, j}^{n, 1}+Y_{\varkappa, j}^{n, 2}-Y_{\varkappa, j}^{n, 3}-Y_{\varkappa, j}^{n, 4} .
\end{aligned}
$$

Next, from (5.17) we directly obtain

$$
\lim _{n \rightarrow \infty}\left\langle\boldsymbol{f}, \boldsymbol{\zeta}_{\varkappa, j}^{n}\right\rangle=\left\langle\boldsymbol{f}, \boldsymbol{\zeta}_{\varkappa, j}\right\rangle,
$$

and thus, comparing limit of (5.12) with (5.1) and by the use of (5.6), (5.9) and (5.17), we can conclude the following

$$
\begin{aligned}
\lim _{n \rightarrow \infty} & \int_{\Omega} \boldsymbol{S}\left(c^{n}, \boldsymbol{D} \boldsymbol{v}^{n}\right) \cdot \boldsymbol{D} \boldsymbol{\zeta}_{\varkappa, j}^{n} d x \\
= & \lim _{n \rightarrow \infty} \int_{\Omega} G_{n}\left(\left|\boldsymbol{v}^{n}\right|^{2}\right)\left(\boldsymbol{v}^{n} \otimes \boldsymbol{v}^{n}\right) \cdot \nabla \boldsymbol{\zeta}_{\varkappa, j}^{n} d x \\
& -\int_{\Omega}(\boldsymbol{v} \otimes \boldsymbol{v}) \cdot \nabla \boldsymbol{\zeta}_{\varkappa, j} d x+\int_{\Omega} \overline{\boldsymbol{S}} \cdot \boldsymbol{D} \boldsymbol{\zeta}_{\varkappa, j} d x=\int_{\Omega} \overline{\boldsymbol{S}} \cdot \boldsymbol{D} \boldsymbol{\zeta}_{\varkappa, j} d x .
\end{aligned}
$$

This together with (5.18) and (4.8) then gives

$$
\lim _{n \rightarrow \infty} Y_{\varkappa, j}^{n, 1}=\int_{\Omega} \overline{\boldsymbol{S}} \cdot\left(\boldsymbol{D} \boldsymbol{\zeta}_{\varkappa, j}+\boldsymbol{D} \boldsymbol{\zeta}_{\varkappa, j}^{2}-\nabla \xi_{\varkappa} \boldsymbol{v}_{j}\right) d x
$$

Let us now investigate the second integral. Using boundedness of $\boldsymbol{S}\left(c^{n}, \boldsymbol{D} \boldsymbol{v}^{n}\right)$ in $L^{\left.\left(p^{n}\right)^{\prime} \cdot \cdot\right)}(\Omega)^{d \times d}$, property (3.2) of the operator $\mathcal{B}$, and zero divergence of $\boldsymbol{v}_{j}^{n}$ on $\left\{x \in \Omega: \boldsymbol{v}^{n}=\boldsymbol{v}_{j}^{n}\right\}$, we can estimate $Y_{\varkappa, j}^{n, 2}$ by the Hölder inequality (and by the definition of the norm) as

$$
\begin{aligned}
Y_{\varkappa, j}^{n, 2} & \leqslant C\left\|\boldsymbol{D} \boldsymbol{\zeta}_{\varkappa, j}^{n, 1}\right\|_{p^{n}(\cdot)} \leqslant C\left\|\operatorname{div} \boldsymbol{v}_{j}^{n} \chi_{\left\{\boldsymbol{v}^{n} \neq \boldsymbol{v}_{j}^{n}\right\}}\right\|_{p^{n}(\cdot)} \leqslant C\left\|\nabla \boldsymbol{v}_{j}^{n} \chi_{\left\{\boldsymbol{v}^{n} \neq \boldsymbol{v}_{j}^{n}\right\}}\right\|_{p^{n}(\cdot)} \\
& \leqslant \frac{C}{2^{j / p^{+}}},
\end{aligned}
$$

where the last inequality follows from (4.13). For the last two integrals $Y_{\varkappa, j}^{n, 3}$ and $Y_{\varkappa, j}^{n, 4}$ we use the convergence (5.8), boundedness of truncation $\boldsymbol{T}_{\varkappa}$ and weak convergence (4.9) to get

$$
\begin{aligned}
\lim _{n \rightarrow \infty} & \left(Y_{\varkappa, j}^{n, 3}+Y_{\varkappa, j}^{n, 4}\right) \\
& =\int_{\Omega} \xi_{\varkappa} \overline{\boldsymbol{S}} \cdot \boldsymbol{T}_{\varkappa}(\boldsymbol{D} \boldsymbol{v}) d x+\int_{\Omega} \xi_{\varkappa} \boldsymbol{S}\left(c, \boldsymbol{T}_{\varkappa}(\boldsymbol{D} \boldsymbol{v})\right) \cdot\left(\boldsymbol{D} \boldsymbol{v}_{j}-\boldsymbol{T}_{\varkappa}(\boldsymbol{D} \boldsymbol{v})\right) d x .
\end{aligned}
$$


All together then gives

$$
\begin{aligned}
& \lim _{n \rightarrow \infty}\left(Y_{\varkappa, j}^{n, 1}+Y_{\varkappa, j}^{n, 2}-Y_{\varkappa, j}^{n, 3}-Y_{\varkappa, j}^{n, 4}\right) \\
& \leqslant \int_{\Omega} \overline{\boldsymbol{S}} \cdot\left(\boldsymbol{D} \boldsymbol{\zeta}_{\varkappa, j}+\boldsymbol{D} \boldsymbol{\zeta}_{\varkappa, j}^{2}-\nabla \xi_{\varkappa} \boldsymbol{v}_{j}-\xi_{\varkappa} \boldsymbol{T}_{\varkappa}(\boldsymbol{D} \boldsymbol{v})\right) d x+\frac{C}{2^{j / p^{+}}} \\
& \quad-\int_{\Omega} \xi_{\varkappa} \boldsymbol{S}\left(c, \boldsymbol{T}_{\varkappa}(\boldsymbol{D} \boldsymbol{v})\right)\left(\boldsymbol{D} \boldsymbol{v}_{j}-\boldsymbol{T}_{\varkappa}(\boldsymbol{D} \boldsymbol{v})\right) d x \\
& =\int_{\Omega} \xi_{\varkappa}\left(\overline{\boldsymbol{S}}-\boldsymbol{S}\left(c, \boldsymbol{T}_{\varkappa}(\boldsymbol{D} \boldsymbol{v})\right)\right) \cdot\left(\boldsymbol{D} \boldsymbol{v}_{j}-\boldsymbol{T}_{\varkappa}(\boldsymbol{D} \boldsymbol{v})\right) d x-\int_{\Omega} \overline{\boldsymbol{S}} \cdot \boldsymbol{D} \boldsymbol{\zeta}_{\varkappa, j}^{1} d x+\frac{C}{2^{j / p^{+}}} .
\end{aligned}
$$

Nevertheless, from the weak lower semicontinuity (see [5]) we also have

$$
\left\|\boldsymbol{D} \boldsymbol{\zeta}_{\varkappa, j}^{1}\right\|_{p(\cdot)} \leqslant \limsup _{n \rightarrow \infty}\left\|\boldsymbol{D} \boldsymbol{\zeta}_{\varkappa, j}^{n, 1}\right\|_{p^{n}(\cdot)} \leqslant \frac{C}{2^{j / p^{+}}},
$$

and thus, we obtain

$$
\begin{aligned}
& \lim _{n \rightarrow \infty}\left(Y_{\varkappa, j}^{n, 1}+Y_{\varkappa, j}^{n, 2}-Y_{\varkappa, j}^{n, 3}-Y_{\varkappa, j}^{n, 4}\right) \\
& \quad \leqslant \int_{\Omega} \xi_{\varkappa}\left(\overline{\boldsymbol{S}}-\boldsymbol{S}\left(c, \boldsymbol{T}_{\varkappa}(\boldsymbol{D} \boldsymbol{v})\right)\right) \cdot\left(\boldsymbol{D} \boldsymbol{v}_{j}-\boldsymbol{T}_{\varkappa}(\boldsymbol{D} \boldsymbol{v})\right) d x+\frac{C}{2^{j / p^{+}}} .
\end{aligned}
$$

Going back to (5.15), we can finally let $j, n, \varkappa \rightarrow \infty$ and estimate

$$
\begin{aligned}
& \lim _{\varkappa \rightarrow \infty} \lim _{j \rightarrow \infty} \lim _{n \rightarrow \infty}\left(J_{\varkappa}^{n}+I_{\varkappa}^{n}\right) \\
& \leqslant \lim _{\varkappa \rightarrow \infty} \lim _{j \rightarrow \infty} \lim _{n \rightarrow \infty}\left(C\left(Y_{\varkappa, j}^{n, 1}+Y_{\varkappa, j}^{n, 2}-Y_{\varkappa, j}^{n, 3}-Y_{\varkappa, j}^{n, 4}+\frac{C(\varkappa)}{2^{j}}\right)^{\frac{1}{4}}|\Omega|^{\frac{3}{4}}+\frac{C}{\sqrt{\varkappa}}+\frac{C}{2^{j}}\right) \\
& \leqslant \lim _{\varkappa \rightarrow \infty} C\left(\left(\int_{\Omega}\left(\overline{\boldsymbol{S}}-\boldsymbol{S}\left(c, \boldsymbol{T}_{\varkappa}(\boldsymbol{D} \boldsymbol{v})\right)\right) \cdot\left(\boldsymbol{D} \boldsymbol{v}-\boldsymbol{T}_{\varkappa}(\boldsymbol{D} \boldsymbol{v})\right) d x\right)^{\frac{1}{4}}+\frac{C}{\sqrt{\varkappa}}\right)=0,
\end{aligned}
$$

where we used point-wise convergence of $\boldsymbol{T}_{\varkappa}(\boldsymbol{D} \boldsymbol{v}) \rightarrow \boldsymbol{D} \boldsymbol{v}$ on $\Omega$ for $\varkappa \rightarrow \infty$ and the Lebesgue dominated convergence theorem. By that we have finished the proof of the claimed (5.14).

5.2. Identification of $\overline{\boldsymbol{S}}$ and $\overline{\boldsymbol{q}}_{c}$. We start with identification of $\overline{\boldsymbol{S}}$. From previous subsection we know that

$$
\lim _{n \rightarrow \infty} \int_{\Omega}\left(\left(\boldsymbol{S}\left(c^{n}, \boldsymbol{D} \boldsymbol{v}^{n}\right)-\boldsymbol{S}\left(c^{n}, \boldsymbol{D} \boldsymbol{v}\right)\right) \cdot\left(\boldsymbol{D} \boldsymbol{v}^{n}-\boldsymbol{D} \boldsymbol{v}\right)\right)^{\frac{1}{4}} d x=0,
$$

which, due to the positiveness of the argument, holds also for a set $Q_{\gamma} \subset \Omega$ such that

$$
Q_{\gamma}:=\{x \in \Omega:|\boldsymbol{D} \boldsymbol{v}| \leqslant \gamma\},
$$

where $\gamma$ is arbitrary positive fixed constant. From the sequence of arguments of integral (5.19), we can find a subsequence (again not relabeled) converging to zero almost everywhere in $Q_{\gamma}$. Then, according to Egoroff theorem, for arbitrary $\varepsilon>0$, we can find a set $Q_{\gamma}^{\varepsilon} \subset \Omega$ which differs from $Q_{\gamma}$ by $\left|Q_{\gamma} \backslash Q_{\gamma}^{\varepsilon}\right|<\varepsilon$, on which the 
sequence of arguments converge uniformly. It is clear, that by the choice of $Q_{\gamma}^{\varepsilon}$, we have

$$
\lim _{\gamma \rightarrow \infty} \lim _{\varepsilon \rightarrow 0}\left|\Omega \backslash Q_{\gamma}^{\varepsilon}\right|=0,
$$

and additionally, from the uniform convergence, that

$$
\lim _{n \rightarrow \infty} \int_{Q_{\gamma}^{\varepsilon}}\left(\boldsymbol{S}\left(c^{n}, \boldsymbol{D} \boldsymbol{v}^{n}\right)-\boldsymbol{S}\left(c^{n}, \boldsymbol{D} \boldsymbol{v}\right)\right) \cdot\left(\boldsymbol{D} \boldsymbol{v}^{n}-\boldsymbol{D} \boldsymbol{v}\right) d x=0 .
$$

Here, due to boundedness of $\boldsymbol{D} \boldsymbol{v}$ on $Q_{\gamma}^{\varepsilon}$ we have $\boldsymbol{S}\left(c^{n}, \boldsymbol{D} \boldsymbol{v}\right) \rightarrow \boldsymbol{S}(c, \boldsymbol{D} \boldsymbol{v})$ strongly in $L^{q}(\Omega)^{d \times d}$ for $q<\infty$, and thus, together with the weak convergence of $\boldsymbol{D} \boldsymbol{v}^{n}$ and strong convergence of $\boldsymbol{S}\left(c^{n}, \boldsymbol{D} \boldsymbol{v}\right)$ in corresponding spaces, we obtain from (5.20)

$$
\lim _{n \rightarrow \infty} \int_{Q_{\gamma}^{\varepsilon}} \boldsymbol{S}\left(c^{n}, \boldsymbol{D} \boldsymbol{v}^{n}\right) \cdot\left(\boldsymbol{D} \boldsymbol{v}^{n}-\boldsymbol{D} \boldsymbol{v}\right) d x=0 .
$$

Hence, using (5.9), we can identify

$$
\lim _{n \rightarrow \infty} \int_{Q_{\gamma}^{\varepsilon}} \boldsymbol{S}\left(c^{n}, \boldsymbol{D} \boldsymbol{v}^{n}\right) \cdot \boldsymbol{D} \boldsymbol{v}^{n} d x=\int_{Q_{\gamma}^{\varepsilon}} \overline{\boldsymbol{S}} \cdot \boldsymbol{D} \boldsymbol{v} d x .
$$

As a next step, we use the monotonicity assumption on $\boldsymbol{S}(1.4)$ guaranteeing the sign of

$$
0 \leqslant \int_{Q_{\gamma}^{\varepsilon}}\left(\boldsymbol{S}\left(c^{n}, \boldsymbol{D} \boldsymbol{v}^{n}\right)-\boldsymbol{S}\left(c^{n}, \boldsymbol{B}\right)\right) \cdot\left(\boldsymbol{D} \boldsymbol{v}^{n}-\boldsymbol{B}\right) d x
$$

where $\boldsymbol{B} \in L^{\infty}\left(Q_{\gamma}^{\varepsilon}\right)^{d \times d}$ is fixed but arbitrary. From (5.21) and the fact that $\boldsymbol{S}\left(c^{n}, \boldsymbol{B}\right) \rightarrow \boldsymbol{S}(c, \boldsymbol{B})$ strongly in $L^{q}(\Omega)^{d \times d}$ for $q<\infty$, we can take the limit $n \rightarrow \infty$ in (5.22) and obtain

$$
\begin{aligned}
0 \leqslant \lim _{n \rightarrow \infty} & \int_{Q_{\gamma}^{\varepsilon}}\left(\boldsymbol{S}\left(c^{n}, \boldsymbol{D} \boldsymbol{v}^{n}\right)-\boldsymbol{S}\left(c^{n}, \boldsymbol{B}\right)\right) \cdot\left(\boldsymbol{D} \boldsymbol{v}^{n}-\boldsymbol{B}\right) d x \\
& =\int_{Q_{\gamma}^{\varepsilon}} \overline{\boldsymbol{S}} \cdot(\boldsymbol{D} \boldsymbol{v}-\boldsymbol{B}) d x-\int_{Q_{\gamma}^{\varepsilon}} \boldsymbol{S}(c, \boldsymbol{B}) \cdot(\boldsymbol{D} \boldsymbol{v}-\boldsymbol{B}) d x \\
& =\int_{Q_{\gamma}^{\varepsilon}}(\overline{\boldsymbol{S}}-\boldsymbol{S}(c, \boldsymbol{B})) \cdot(\boldsymbol{D} \boldsymbol{v}-\boldsymbol{B}) d x .
\end{aligned}
$$

Choosing $\boldsymbol{B}=\boldsymbol{D} \boldsymbol{v} \pm \lambda \boldsymbol{A}(x)\left(\lambda>0\right.$ and $\left.\boldsymbol{A} \in L^{\infty}\left(Q_{\gamma}^{\varepsilon}\right)^{d \times d}\right)$, we can due to the continuity of $\boldsymbol{S}$ in $\boldsymbol{B}$ identify

$$
\overline{\boldsymbol{S}}=\boldsymbol{S}(c, \boldsymbol{D} \boldsymbol{v}) \text { a.e. on } Q_{\gamma}^{\varepsilon} .
$$

To finish the proof, we extend this result on the whole domain $\Omega$ by letting $\varepsilon \rightarrow 0$ and then $\gamma \rightarrow \infty$. Consequently, using strict monotonicity (1.5), we conclude that $\boldsymbol{D} \boldsymbol{v}^{n} \rightarrow \boldsymbol{D} \boldsymbol{v}$ a.e. in $\Omega$. By that, together with (5.7), we can identify $\overline{\boldsymbol{q}}_{c}$, which brings the proof of Theorem 1 to the end.

\section{REFERENCES}

[1] E. Acerbi and N. Fusco. An approximation lemma for $W^{1, p}$ functions. In J. M. Ball, editor, Material Instabilities in Continuum Mechanics and Related Mathematical Problems, pages 1-5, Oxford, 1988. Oxford University Press. 
[2] A. Bensoussan and J. Frehse. Regularity results for nonlinear elliptic systems and applications, volume 151 of Applied mathematical sciences. Springer-Verlag, Berlin-Heidelberg, 2002.

[3] D. Brooks, J. Goodwin, and G. Seaman. Interactions among erythrocytes under shear. J Appl Physiol, 28(2):172-177, 1970.

[4] M. Bulíček, J. Málek, and K. Rajagopal. Mathematical Results Concerning Unsteady Flows of Chemically Reacting Incompressible Fluids. In J. C. Robinson and J. L. Rodrigo, editors, Partial Differential Equations and Fluid Mechanics, volume 7, pages 26-53. Cambridge University Press, London, 2009.

[5] M. Bulíček and P. Pustějovská. On Existence analysis of steady flows of generalized Newtonian fluids with concentration dependent power-law index. Journal of Mathematical Analysis and Applications, 402(1):157-166, 2013.

[6] D. Cruz-Uribe, A. Fiorenza, and C. Neugebauer. The maximal function on variable $L^{p}$ spaces. Annales Academiae Scientiarum Fennicae Mathematica, 28:223-238, 2003.

[7] G. Dal Maso and F. Murat. Almost everywhere convergence of gradients of solutions to nonlinear elliptic systems. Nonlinear Anal., 31(3-4):405-412, 1998.

[8] E. De Giorgi. Sulla differenziabilitae l'analiticita delle estremali degli integrali multipli regolari. Mem. Accad. Sci. Torino. Cl. Sci. Fis. Mat. nat., 3:25-43, 1957.

[9] L. Diening. Maximal function on generalized Lebesgue spaces $L^{p(\cdot)}$. Mathematical Inequalities \& Applications, 7(2):245-253, 2004.

[10] L. Diening, P. Harjulehto, P. Hästö, and M. Růžička. Lebesgue and Sobolev spaces with variable exponents, volume 2017 of Lecture Notes in Mathematics. Springer, Heidelberg, 2011.

[11] L. Diening, P. Hästö, and A. Nekvinda. Open problems in variable exponent lebesgue and sobolev spaces. In Drabek and Rakosnik, editors, FSDONA04 Proceedings, pages 38-58, 2005.

[12] L. Diening, C. Kreuzer, and E. Süli. Finite element approximation of steady flows of incompressible fluids with implicit power-law-like rheology. SIAM Journal on Numerical Analysis, 51(2):984-1015, 2013.

[13] L. Diening, J. Málek, and M. Steinhauer. On Lipschitz truncations of Sobolev functions (with variable exponent) and their selected applications. Control, Optimisation and Calculus of Variations, 14(2):211-232, Mar. 2008.

[14] X. Fan, J. Shen, and D. Zhao. Sobolev Embedding Theorems for Spaces $W^{k, p(x)}(\Omega)$. Journal of Mathematical Analysis and, 262(2):749-760, 2001.

[15] X. Fan, S. Wang, and D. Zhao. Density of $C^{\infty}(\Omega)$ in $W^{1, p(x)}(\Omega)$ with discontinuous exponent $p(x)$. Mathematische Nachrichten, 279(1-2):142-149, Jan. 2006.

[16] J. Frehse, J. Málek, and M. Steinhauer. An Existence Result for Fluids with Shear Dependent Viscosity - Steady Flows. Nonlinear Analysis: Theory, Methods \& Applications, 30(5):3041-3049, 1997.

[17] J. Frehse, J. Málek, and M. Steinhauer. On analysis of steady flows of fluids with shear-dependent viscosity based on the Lipschitz truncation method. SIAM J. Math. Anal., 34(5):1064-1083, 2003.

[18] G. Galdi, R. Rannacher, A. Robertson, and S. Turek. Hemodynamical flows: modeling, analysis and simulation, volume 37. Birkhäuser, Basel, Boston, Berlin, 2008 . 
[19] P. Hästö. Counter-examples of regularity in variable exponent Sobolev spaces. In The p-Harmonic Equation and Recent Advances in Analysis, volume 370 of Contemporary Mathematics. American Mathematical Society, 2005.

[20] J. Hron, J. Málek, P. Pustějovská, and K. R. Rajagopal. On the Modeling of the Synovial Fluid. Advances in Tribology, 2010(Art. No. 104957), 2010.

[21] O. Kováčik and J. Rákosník. On spaces $L^{p(x)}$ and $W^{k, p(x)}$. Czechoslovak Math. $J, 41(116): 592-618,1991$.

[22] J. Musielak. Orlicz Spaces and Modular Spaces. Springer-Verlag, Berlin, 1983.

[23] H. Nakano. Modulared semi-ordered linear spaces, volume 1 of Tokyo mathematical book series. Maruzen Co., Tokyo, 1950.

[24] J. Nash. Continuity of solutions of parabolic and elliptic equations. American Journal of Mathematics, 80(4):931-954, 1958.

[25] W. Orlicz. Über konjugierte Exponentenfolgen. Stud. Math., 3:200-211, 1931.

[26] L. Pick and M. Růžička. An Example of a Space $L^{p(x)}$ on which the HardyLittlewood Maximal Operator is not Bounded. Expositiones Mathematicae, 19(201):369-371, 2001.

[27] P. Pustějovská. Biochemical and mechanical processes in synovial fluid - modeling, analysis and computational simulations. Phd thesis, Charles University in Prague, 2012.

[28] K. Rajagopal and M. Růžička. On the Oberbeck Boussinesq approximation. Mathematical Models and, 1996.

[29] M. Růžička. Electrorheological fluids: modeling and mathematical theory, volume 1748 of Lecture Notes in Mathematics. Springer-Verlag, Berlin-Heidelberg, 2000.

[30] M. Růžička. Modeling, mathematical and numerical analysis of electrorheological fluids. Appl. Math, 49:565-609, 2004.

[31] I. Sharapudinov. On the topology of the space $L^{p(t)}([0,1])$. Matem. Zametki, 26:613-632, 1978.

Mathematical Institute, Charles University in Prague, Sokolovská 83, 18675 Prague 8, Czech Republic

E-mail address: mbul8060@karlin.mff.cuni.cz

Institute of Computational Mathematics, Graz University of Technology, SteyrERgasse 30, A-8010 Graz, Austria

E-mail address: pustejovska@tugraz.at 